\title{
Future scope and expectations: why, when, and how LECs should continue
}

\author{
W. CAIRNS S. SMITH \\ Department of Public Health, University of Aberdeen, Foresterhill, \\ Aberdeen AB9 2ZD
}

Accepted for publication 25 August 1999

\begin{abstract}
Summary There is a strong case to continue to use LEC approaches, as they are a comprehensive and cost effective means of delivering the key elements of leprosy control. LECs should be conducted when there is evidence of large numbers of hidden cases. Probably a minimum of two LECs is required but where large number of new cases continue to be detected they could be run on an annual basis. The methodology of LECs needs to be improved through experience, evaluation and from LECs conducted elsewhere; feedback from the community is also important. There is room to improve all aspects of LECs: planning, training, education, diagnosis and treatment completion.
\end{abstract}

\section{Introduction}

Leprosy Elimination Campaigns (LEC) were introduced with three basis elements: capacity building for local health workers, to increase community participation, and to diagnose and treat leprosy. In practice, LECs have had many additional effects such as promoting community awareness, reduction in stigma, and improved accessibility of MDT. Initially the LECs were implemented in populations of around 0.5 million, but these have now ranged in population coverage up to country level campaigns.

This discussion paper attempts to address some fundamental questions about the future of LECs and their role in leprosy programmes. The first question is why should they continue or whether they should continue at all. If they are to continue, we need to consider when and where they should be implemented. Finally, on the basis of experiences with the LECs to date, we need to address questions about how they should be conducted.

\section{Why should LECs continue?}

ECONOMIC APPRAISAL

This is the most fundamental and challenging question and needs to be asked. This can be addressed from an economic point of view in terms of a cost-benefit analysis. What are the 
costs of a LEC and are the benefits worth the cost? In considering the costs, we should include patient costs and programme costs, as well as opportunity costs. If we did not use our resources to conduct LECs, we could use them in other ways, either in leprosy programmes or even in other health programmes. There is an opportunity cost for local health workers in terms of what they forgo in order to spend time in LEC activities. Similarly, in considering the benefits, we can look wider than the achievements of the three principal objectives and look at added value to the health care system, other programmes which can benefit from the activities and the sustainability and long-term effects of the intervention.

This economic approach involves value judgements about the costs and benefits of LECs. However it also leads us to review the potential within LECs of minimizing costs and maximizing benefits. This perhaps is more about the 'how' than the 'why' of LECs, but does suggest that we can tip the balance as to whether to continue with LECs or not, by minimizing costs (through combinations with other programmes, joint training and media opportunities) and by maximizing the benefits (by being sustainable, contributing to epidemiological monitoring, and strengthening health care infrastructure).

\section{OPTIONS APPRAISAL}

LECs have an opportunity costs, but what are the opportunities which we forgo by utilizing our resources, both funding and time, on LECs? What options should we be considering to achieve the same goals?

The first objective is in capacity building for local health workers to improve MDT services. These could be improved by ensuring that leprosy was included in training and retraining curricula, or by running specialized training courses. The second objective is in increasing community participation that could be tackled in other ways as part of community development programmes. Actual detection and treatment could be improved by further strengthening of the health care infrastructure.

The objectives of LECs can be achieved in other ways. However, LECs combine activities into one activity in a cost-effective way with important interactions.

\section{EPIDEMIOLOGICAL AND CONTROL OBJECTIVES}

It could be argued that the information provided by the LECs assists in the assessment and monitoring of the leprosy situation. However, the information derived in this way needs to treated with caution. LECs would, on their own, provide epidemiological information if they were conducted at regular intervals using identical methods, but this is never likely to be the case as the methods are tailored to the evolving circumstances. LECs can provide useful information but this needs to be interpreted with information from other sources.

LECs may be considered as achieving the aims of elimination but elimination, that is prevalence reduction, is only part of the achievements of LECs. LECs contribute to potential eradication as well as community education and sustainable capacity building.

\section{When should LECs be undertaken?}

LECs should be undertaken when there are significant numbers of undetected cases in a population-hidden cases. This requires information about the disease and the health-care 
inf rastructure. The situations suitable are ones where there is a gap between the registered and estimated cases. The methods for estimating cases are relevant to this task. The classification pattern and disability rates in new cases can provide some clues to the completeness of case detection and the likely burden of undetected cases.

The basis that there is a requirement for expecting large numbers of hidden cases requires high prevalence rates, large populations or both together. The level at which LECs are conducted will depend on disease distribution, political boundaries, population size, languages and the administrative organization of the health service.

The other factor in timing is to consider the frequency of implementing LECs. A LEC which results in few new cases detected could be due to either poorly conducted LECs or that the estimate of hidden cases was wrong. There is a case for conducting LECs more than once to resolve this question, reviewing the methods of implementing the LEC. This could be on annual basis or every 2 years. In the past, surveys were often conducted every 5 years, so that a 2-year gap could be acceptable.

The case for an annual LEC can be made in that a routine is established, regular budgets can be committed and political commitment sustained. LEC methods can be improved from the experience of previous LECs. LECs that repeatedly produce few new cases can be stopped as indicating that there are unlikely to be significant numbers of hidden cases.

\section{How should LECs be conducted in the future?}

There is room for improvement in all aspects of LECs from planning through to evaluation.

Planning is a key part to the process and the experience of successive LECs should improve this process which includes preparation of media materials, drug distribution and trainers.

LECs must involve the general health services at all levels; this is crucial for sustainability and treatment completion.

Quality of diagnosis must be kept high, limiting both over-diagnosis and under-diagnosis. Both are inevitable, but the size should be contained. A degree of over-diagnosis is more acceptable than under-diagnosis and missing of cases. However, repeated LECs can pick up missed cases.

Treatment completion rates need not be $100 \%$, but it is important that these are monitored and acceptable levels achieved.

The recent LEAG meeting made recommendations about the core elements of lepros control activities that listed eight points. All eight of the core points are areas that are addressed within LECs (accessibility, drug supply, monitoring, commitment, education, special populations, integration and prevention of disabilities. In this sense, LECs deliver the core elements of leprosy control programmes). It is important that these core elements are maintained with in future LECs. 
Appendix: results of Western Pacific Region WHO leprosy elimination programme

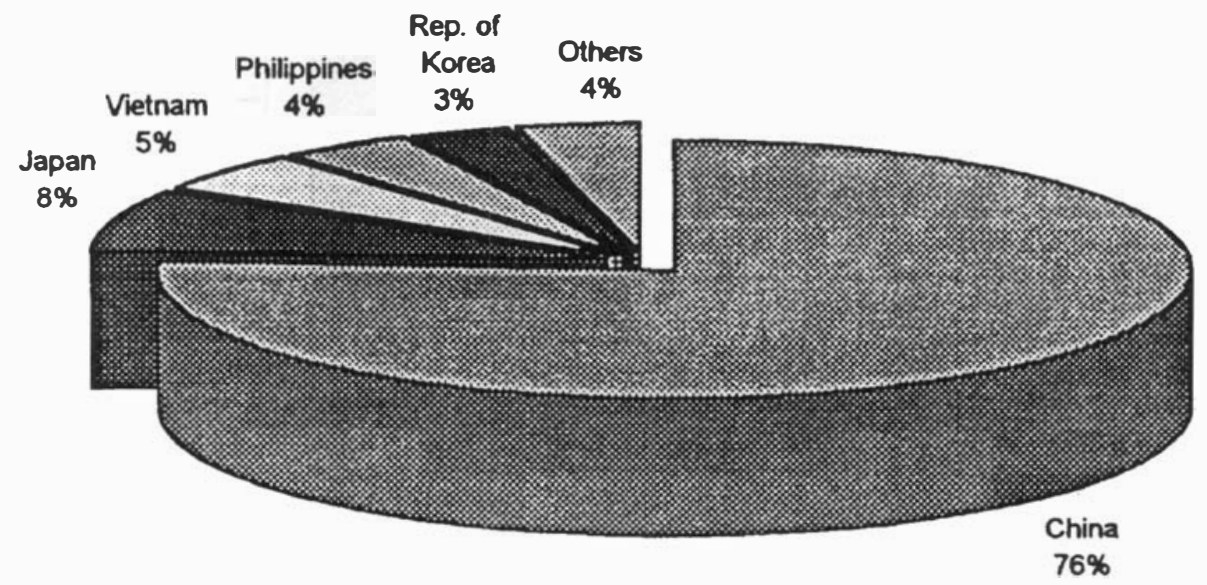

Total estimated population-1653 million

Diagram 1. Population distribution by major countries, 1998.

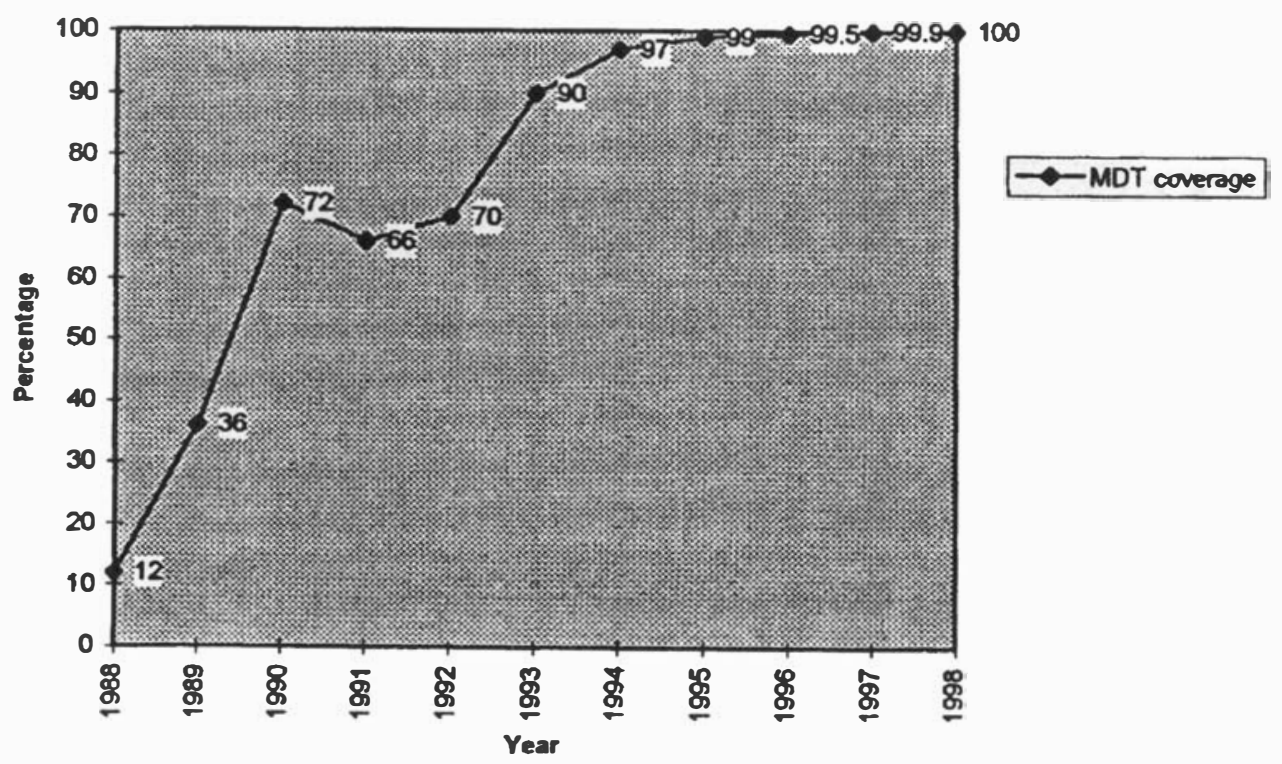

Diagram 2. MDT coverage, 1988-1998. 


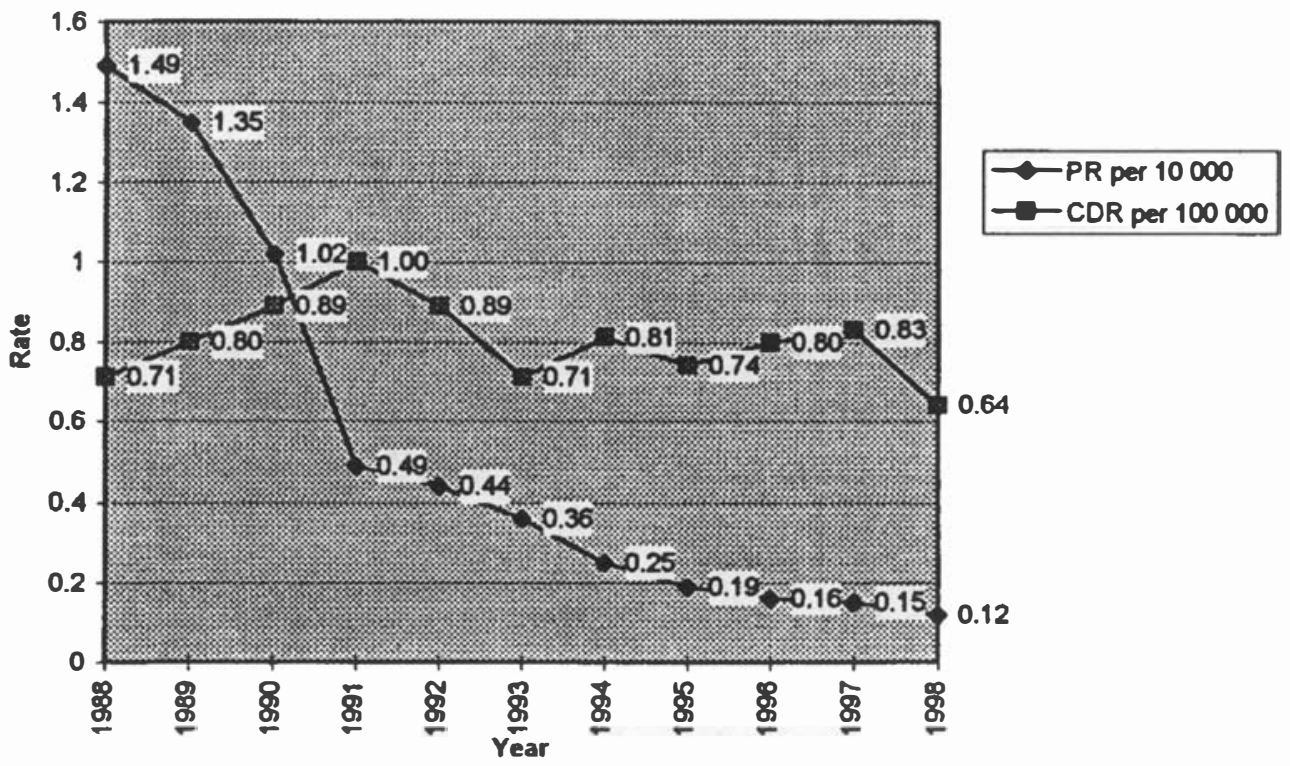

Diagram 3. Prevalence and case detection rates, 1988-1998.

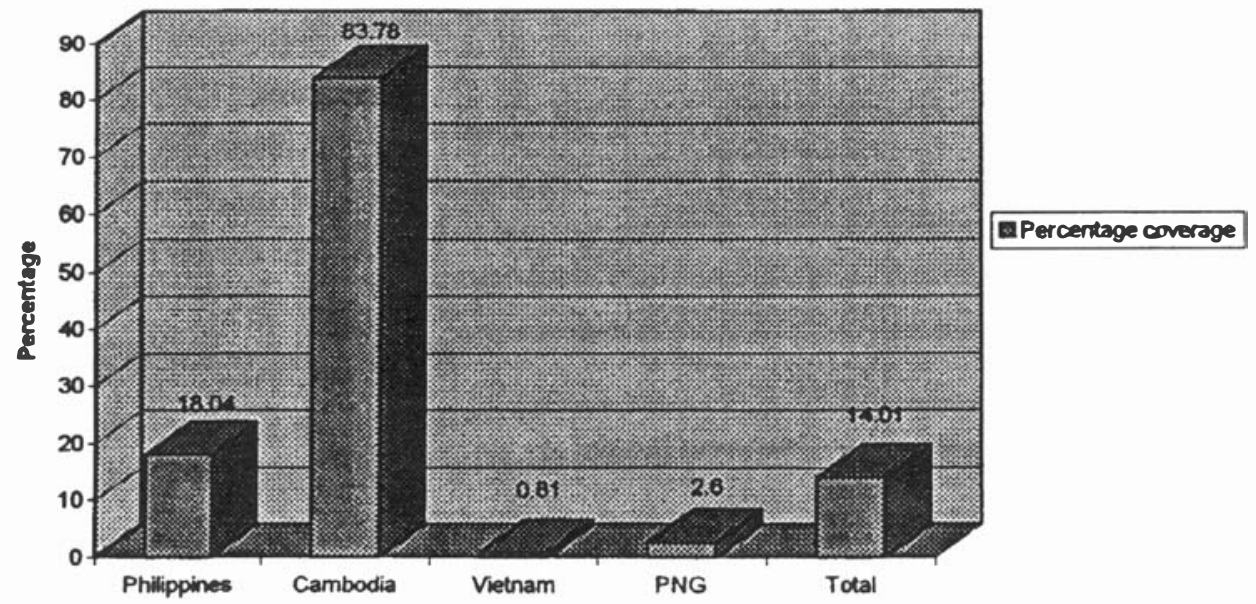

Diagram 4. Proportion of LEC project population to country population, 1996-1998. 


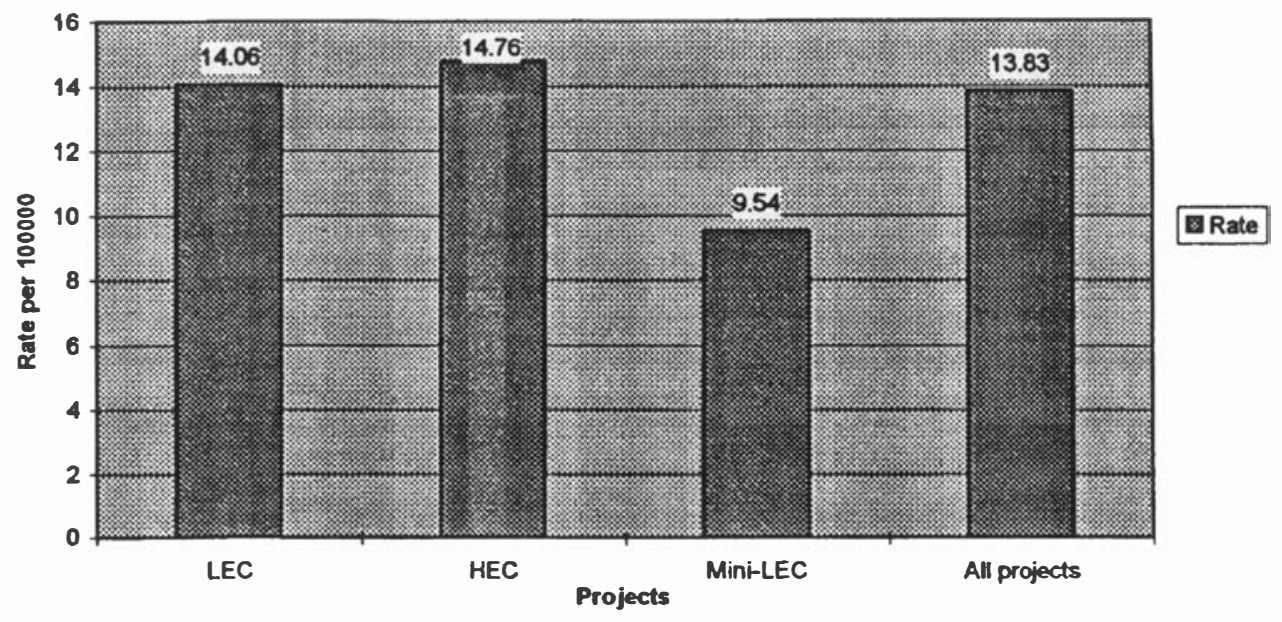

Diagram 5. Case detection rate by type of project, 1996-1998.

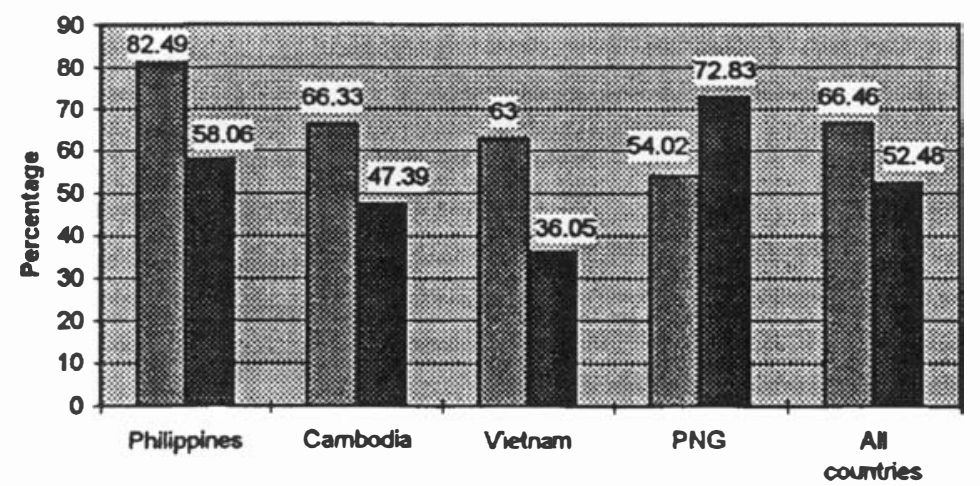

\section{MB proporition Routine}

MB proportion Project

Diagram 6. Average proportion of $\mathrm{MB}$ among new cases during project period and project years by country, 1996-1998

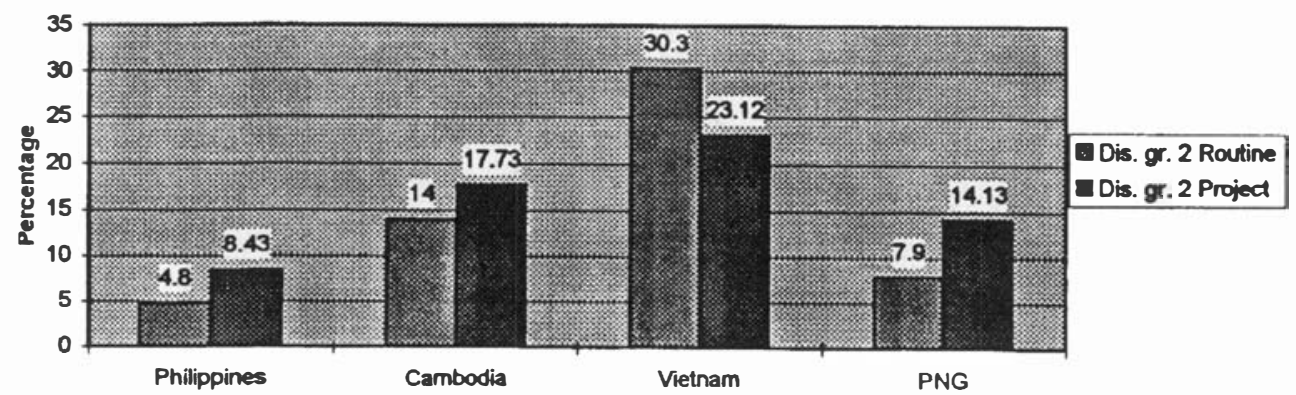

Diagram 7. Average proportion of disability grade 2 among new cases during project period and project years, 1996-1998. 


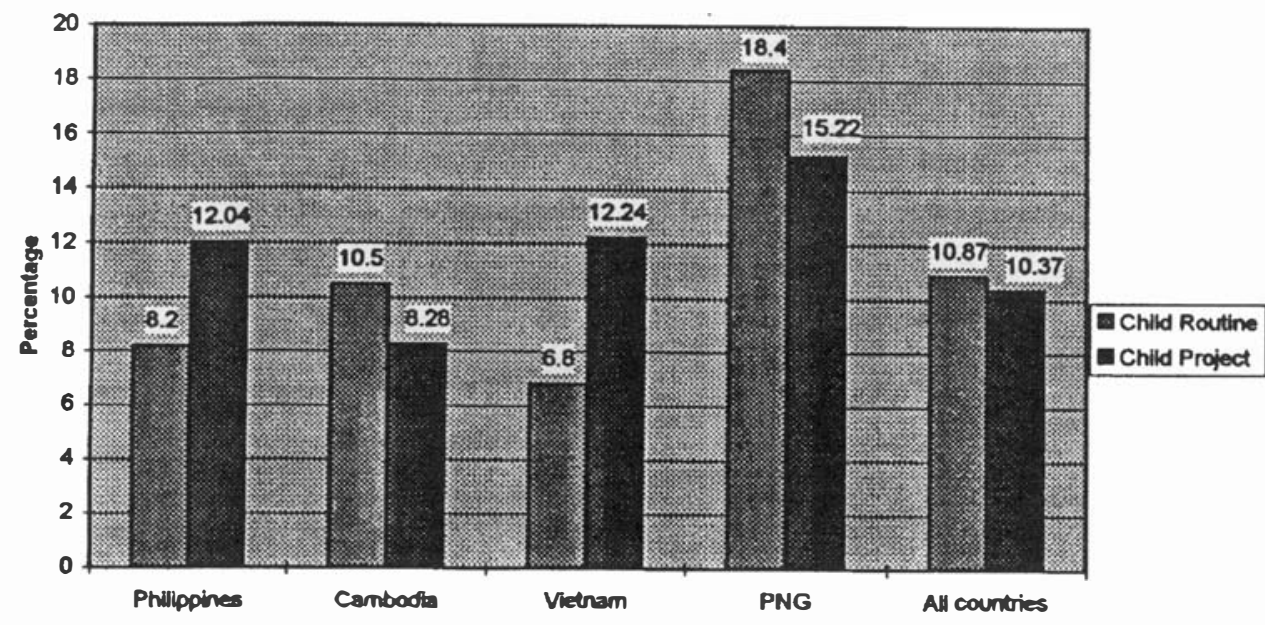

Diagram 8. Average proportion of child cases among new during project period and project years, 1996-1998.

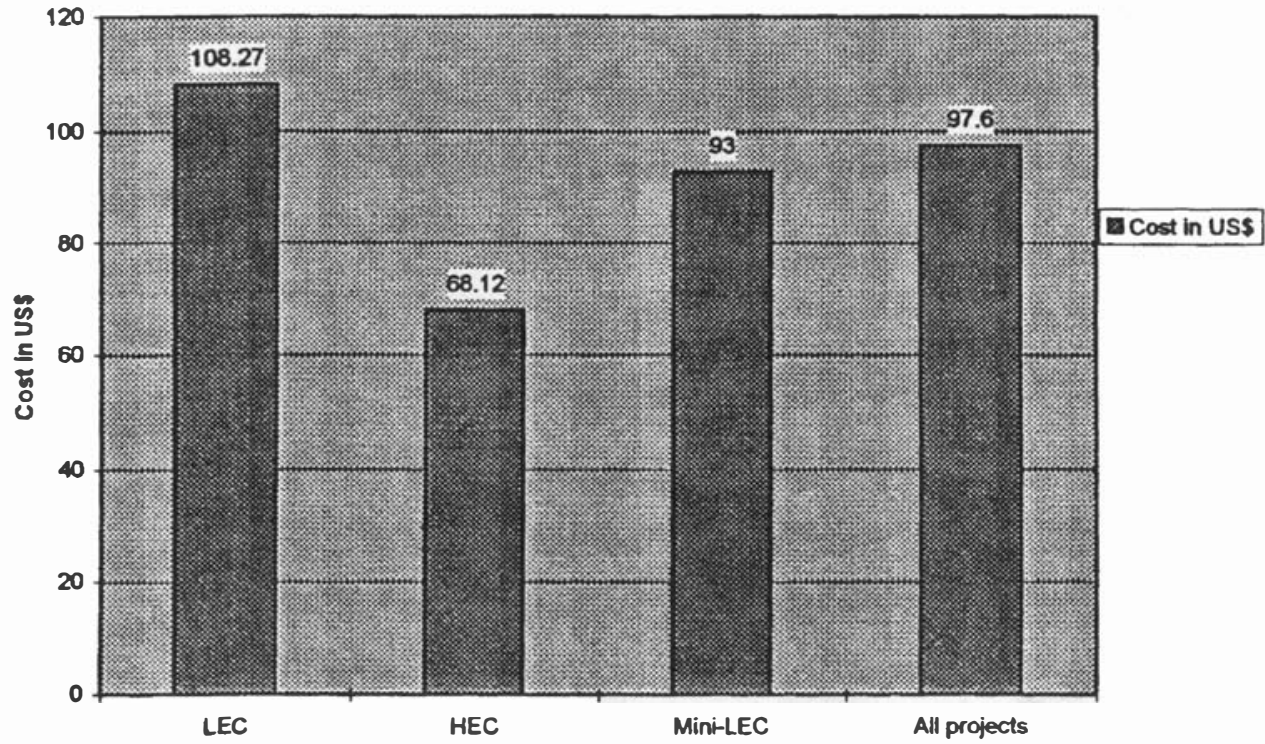

Diagram 9. Average cost per case detected in US\$ by project, 1996-1998. 


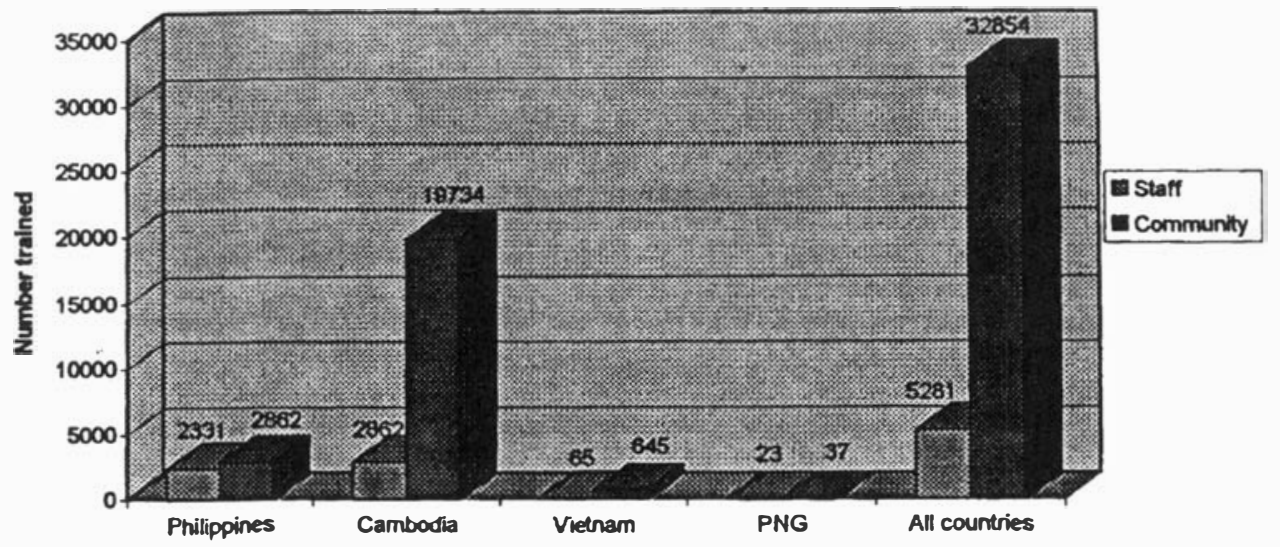

Diagram 10. Number of staff and community trained by countries, 1996-1998. 